\section{La necesidad de una regulación legal de la investigación amigable a la demencia y otros trastornos cognitivos}

\author{
The need of a dementia-friendly legal \\ regulation of researching
}

\section{Señor Editor:}

El relevante aumento del número de personas con trastornos cognitivos y demencias establece desafíos y dilemas en la investigación que incorpora a estos grupos desde la regulación legal y bioética. Las personas con trastornos cognitivos y demencias son frecuentemente excluidas de investigación debido, entre otros motivos, con estigma que lleva a la asunción naturalizada de incapacidad total para consentir o de informar válidamente ${ }^{1}$, lo que resulta contradictorio con las declaraciones existentes sobre la urgencia de aumentar investigación científica que permita una mejor comprensión de las implicancias sociales y clínicas de los trastornos cognitivos y demencias, emanadas entre otros, por la Organización Mundial de la Salud ${ }^{2}$ y el bloque G8 a través del informe emanado luego de la Cumbre de Acción Global contra la demencia, realizada en el año 2013.

En Chile, la ley 20.120 establece que toda investigación científica realizada con seres humanos deberá contar con el consentimiento previo escrito, expreso, libre, informado y personal, pudiendo entonces reconocerse esta acción como la aproximación concreta a la capacidad de expresión de voluntad en las personas según dicha ley.

Por otra parte, la ley 20.584 que regula los derechos y deberes de las personas en relación con acciones vinculadas a su atención en salud, alude directamente a los derechos de las personas con discapacidad psíquica e intelectual, refiriendo en su artículo 28 que ninguna persona con discapacidad psíquica o intelectual que no pueda expresar su voluntad podrá participar en una investigación científica y que en los casos en que se realice investigación científica con participación de personas con discapacidad psíquica o intelectual que tengan la capacidad de manifestar su voluntad y que hayan dado consentimiento informado, además de la evaluación ética que corresponda, será necesaria la autorización de la autoridad sanitaria competente, además de la manifestación de voluntad expresa de participar tanto de parte del sujeto como de su representante legal.

Al respecto, es necesario reflexionar sobre el significado e implicancias detrás de las ideas planteadas en el marco legal y que no son explicitadas en éste. ¿Qué se entiende por capacidad de expresar la voluntad? ¿Se relaciona en algún nivel con la capacidad de llevar a cabo un proceso de consentimiento informado personal y escrito, o responde a otra categoría de capacidades personales? ¿Cómo se comprende la capacidad de consentir? Si una persona con discapacidad psíquica puede manifestar su voluntad, ¿por qué requiere de un representante legal? A una primera mirada, puede interpretarse que dichos procesos se centran en las competencias cognitivas individuales involucradas, en atemporalidad, descontextualización y sin la implicancia de terceros como parte de dicho constructo denominado expresión de voluntad.

Del mismo modo, resulta oportuno reflexionar sobre la pertinencia del consentimiento informado según su caracterización en las normativas legales en nuestro país como herramienta central de la definición de inclusión de participación de personas con trastornos cognitivos, discapacidad psíquica e intelectual. ¿Es ésta una herramienta suficiente para garantizar protección sin promover la exclusión por el hecho de vivir con un trastorno cognitivo o demencia? ¿Podría considerarse el uso de modalidades no necesariamente basadas en la lectoescritura como acercamiento a la expresión de voluntad de las personas?

¿Quién debiese adaptarse para acceder a la expresión de voluntad: las personas o los instrumentos que de manera consensuada y racional seleccionamos para estudiar la realidad?

Se ha descrito que la noción de competencia para una investigación de orden biomédico tiene tres elementos cognitivos fundamentales: la comprensión de la información sobre lo que involucra participar en una investigación, el procesamiento de dicha información, y la comunicación adecuada y clara de una decisión sobre participación en una determinada investigación ${ }^{3}$. Un caso a considerar es 
el de la enfermedad de Alzheimer en etapa leve, ya que se ha referido que sus características clínicas y el déficit cognitivo implicado no involucra déficits significativos en las tres áreas cognitivas previamente referidas y particularmente la habilidad de comprender y codificar información no experimenta déficit relevante debido a que las dificultades semánticas y atencionales son aún controlables ${ }^{4}$.

Desde una mirada más estructural, se ha sugerido observar críticamente los contextos legales y normativos que regulan la investigación, propendiendo a posibilitar la protección de la autonomía y la voluntariedad de los sujetos con discapacidad psíquica e intelectual; y garantizar el derecho a participar en investigación comprendiendo el beneficio potencial que ésta debe tener para las perso- nas ${ }^{5}$. Esta necesidad es también congruente con lo planteado en la declaración Universal de Derechos Humanos por medio del derecho a la participación social y la satisfacción de necesidades de seguridad.

¿Cómo puede incluirse a personas con trastornos cognitivos y demencia en investigación, garantizando su protección y promoviendo su ejercicio de autonomía? La organización Alzheimer's Association elaboró una serie de recomendaciones dirigidas a investigadores, comités de bioética y revisores científicos, sobre la inclusión de personas con problemas cognitivos, sugiriendo estrategias concretas para la promoción de estos procesos y guiar la elaboración y aplicación de consentimiento informado en personas con demencia y trastornos cognitivos ${ }^{5}$, las que son sintetizadas a continuación (Tabla 1).

Tabla 1. Recomendaciones sobre la inclusión de personas con problemas cognitivos en investigación científica

\section{Tema}

Principios bioéticos involucrados

Situaciones en las que personas que no pueden consentir por discapacidad cognitiva pueden ser involucradas en investigación

Situaciones en las que personas que no pueden consentir por discapacidad cognitiva no debiesen ser involucradas en investigación

Evaluación inicial de capacidad para consentir

Capacidad de consentir en relación con alteración cognitiva

Incapacidad de consentir

\section{Recomendación}

La discapacidad cognitiva no siempre se asocia con la falta de capacidad para llevar a cabo un proceso de consentimiento informado. La exclusión de una investigación de alguna persona que sea elegible por el hecho aislado de tener discapacidad cognitiva, ya sea que carezca o no de capacidad para consentir su participación en la investigación, es discriminatorio y atenta contra el principio bioético de justicia

a. El investigador ofrece un potencial razonable de beneficio directo relacionado con salud que puede incluir la calidad de vida; si no hay un potencial de beneficio directo relacionado con salud para el individuo, entonces la investigación debe plantear riesgos mínimos para la persona de acuerdo con los comités de bioética y la investigación debe orientarse a conocimiento generalizable sobre la condición o trastorno del participante; b. El consentimiento informado para investigación es completado por quien, bajo ley, tiene la autoridad legal para tomar dichas decisiones por la persona. Si otras personas con relaciones cercanas al individuo con discapacidad cognitiva tienen disponibilidad, es éticamente apropiado consultar a éstas para que provean consentimiento subrogado en relación con la participación

La investigación presenta un riesgo mayor al mínimo y no ofrece un potencial razonable de beneficio relacionado con salud resultante directo de los procedimientos de investigación

La investigación con personas que presentan discapacidad cognitiva debe considerar en su proceso, una etapa de evaluación inicial que permita identificar aquellos individuos que presenten un riesgo o sospecha de incapacidad para consentir

No debe confundirse la evaluación de alteración cognitiva con la evaluación de capacidad para consentir

La capacidad de consentir debe ser evaluada según las características y requerimientos que cada proyecto de investigación supone para la persona

En caso que la capacidad de consentimiento no sea la apropiada, puede considerarse alguna de las siguientes acciones: asentimiento, identificación de una persona que actúe como acompañante o proxy 
A partir de lo planteado y desde la observación del contexto legal para la investigación en nuestro país, la regulación legal de la investigación en personas con trastornos cognitivos debiese no sólo velar por la protección de estos u otros grupos de personas en vulnerabilidad por medio de la exclusión oportuna, sino que también debe ser propositiva en la formulación de adecuaciones necesarias de los instrumentos y las regulaciones legales y normativas de la relación investigadorparticipante, que facilite su inclusión en un contexto basal de protección.

Es importante resaltar que a pesar de la asociación que se da entre los trastornos cognitivos y la alteración de competencia, dichos diagnósticos no son sinónimos de incompetencia total debido a que son entidades dinámicas, individualizadas y frecuentemente progresivas ${ }^{6}$.

De este modo, se vuelve necesario promover la revisión y estudio de los elementos legales, normativos y clínicos que regulan actualmente la investigación en nuestro país, con el propósito de desarrollar procesos que garanticen la protección y posibiliten la inclusión de personas con trastornos cognitivos, bajo la premisa de la investigación científica como un medio para la generación de conocimiento teórico y práctico que aporte a las personas.

\section{Referencias bibliográficas}

1. Garand L, Lingler J, Conner K, Dew MA. Diagnostic Labels, Stigma, and Participation in Research Related to Demencia and Mild Cognitive Impairment. Res Gerontol Nurs 2009; 2 (2): 112-21.

2. Organización Mundial de la Salud. Dementia: A Public Health priority. 2012.

3. Alexander M. Clinical Determination of Mental Competence: A Theory and a Retrospective Study. JAMA Neurol 1988; 45 (1): 23-6.

4. Gauthier S, Leuzy A, Racine E, Rosa-Neto P. Diagnosis and management of Alzheimer's disease: Past, present and future ethical issues. Prog Neurobiol 2013; 1-12.

5. Alzheimer's Association. Research Consent for Cognitively Impaired Adults: Reccomendations for Institutional Review Boards and Investigators. Alzheimer Dis Assoc Disord 2004; 18 (3): 171-5.

6. Marson DC. Loss of competency in Alzheimer's disease: conceptual and psychometric approaches. Int J Law Psychiatry 2001; 24: 267-83.

Jean Gajardo J. ${ }^{1}$

Centro Kintun para personas con demencia, Servicio Nacional del Adulto Mayor y Municipalidad de Peñalolén. Departamento de Terapia Ocupacional y Ciencia de la Ocupación, Facultad de Medicina, Universidad de Chile.

${ }^{1}$ Terapeuta Ocupacional. Máster en Gerontología Social. Candidato a Doctor en Salud Pública, Escuela de Salud Pública, Universidad de Chile.

Correspondencia:

Jean Gajardo J.

Independencia 1027, 8380453, Independencia,

Santiago.

Teléfono celular: 0991352427

Teléfono fijo: 26322741

E-mail: jean.gajardo@gmail.com 